\title{
Maximizing the value of cryoEM in drug discovery
}

\author{
C Hong ${ }^{1}$ \\ ${ }^{1}$ Merck \& Co., Inc., Kenilworth, NJ, USA \\ chuan.hong@merck.com
}

For decades, protein structures have helped to understand the mechanism of action and facilitate the design of drug candidates. The rapid development of cryo-EM has provided unprecedented opportunities for obtaining structural knowledge for challenging targets, such as membrane proteins and multi-protein complexes. We will describe the approach taken to implement cryo-EM in a pharmaceutical environment. We will then show how cryo-EM structures have been used in drug discovery programs, including modalities such as small molecules, peptides and antibodies. Finally, we will discuss the current challenges and future outlook for increasing the impact of cryoEM in pharmaceutical research and development.

Acta Cryst. (2020). A76, a96 